normale, les résultats portent peut-être sur des chiffres trop petits pour donner une précision suffisante.

La méthode préconisée serait done peut-être applicable aux fromages quand ceux-ci sont fabriqués avec des mélanges de laits entiers. Il serait par ailleurs possible d'empêcher l'addition de lait écrémé de vache en fixant un pourcentage de matière grasse dans l'extrait sec assez éleyé.

\title{
CONCLUSION.
}

Le travail que nous avons l'honneur de vous soumettre n'est qu'une étude préparatoire. Nos essais ne sont pas encore assez nombreux pour nous permettre de donner des conclusions définitives. Mais ils sont cependant assez encourageants pour nous inciter à continuer des recherches plus complètes portant sur un grand nombre d'échantillons. Nous pourrons ainsi fixer des maxima et des minima aux différents indices.

Depuis que nous avons entrepris ces recherches, nous avonslu dans "Le Lait " qu'un travail analogue avait été effectué par M. Lopez (An. Soc. Espanola Fis. Quim. t. XXXII, p. 105-107 et p. 872875 ; - in Le Lait, année 1936, p. 536 et 1122). Ses conclusions sont les mêmes que les nôtres. M. Lopez indique, en outre, une méthode de détermination de l'indice eaprylique, qui permet d'opérer sur une petite quantité de matière grasse et que nous nous proposons d'expérimenter.

\section{L'IDENTIFICATION DU LAIT NEUTRALISÉ (1)}

\section{par}

\section{R. BAETSLÉ}

Directeur du Laboratoire de Chimie et de Bactériologie de la ville de Gand

L'addition de carbonate ou de bicarbonate de sodium au lait a pour but de neutraliser l'acidité lactique au fur et à mesure qu'elle se développe et d'en combattre ainsi les mauvais effets, car, lorsque l'acidité du lait atteint $20 \mathrm{~cm}^{3} \mathrm{~N} / 10$ pour $100 \mathrm{~cm}^{3}$, l'ébullition peut déjà provoquer la coagulation (le maximum tolérable est situé à $22,5 \mathrm{~cm}^{3} \mathrm{~N} / 10$ pour $100 \mathrm{~cm}^{3}$ ou $\left.9 \mathrm{~cm}^{3} \mathrm{~N} / 4\right)$.

La loi belge (arrêté royal du 31 mars 1925, modifié par celui du 25 novembre 1925) déclare nuisible, par application de l'article 561, paragraphe 2, du Code Pénal, le lait additionné d'eau ou de substances étrangères telles qu'agents de conservation, neutralisants,

(1) IVe Congrès international technique et chimique des industries agricoles, Bruxelles 1935. Volume III. Division des Etudes industrielles-Communications et discussions, p. 550 . 
colorants ou épaississants ; elle interdit, par conséquent, d'exposer en vente, de vendre ou de débiter du lait additionné de carbonate ou de bicarbonate de sodium; un tel lait est donc un produit falsifié.

C'est avec raison que la loi belge interdit cette addition, ear le carbonate et le bicarbonate de sodium ne produisent pas seulement une neutralisation de l'acidité du lait, ils en changent profondément la composition naturelle; c'est ainsi qu'ils solubilisent une petite quantité de protéines, ils saponifient des acides gras, provoquent un léger mouillage, agissent sur les phosphates de calcium et de magnésium, donnent des carbonates insolubles et du phosphate de sodium, abaissent le taux butyreux, élèvent le poids spécifique, modifient le pouvoir rotatoire et, ce qui est plus grave, favorisent le développement microbien de façon qu'il n'est plus possible au lait de résister naturellement contre les bactéries qui s'y développent avec une extrême rapidité. Соммény a nettement établi que la «pullulation microbienne au sein du lait ainsi alcalinisé est considérable ; l'ordre de l'accélération du développement microbien du lait bicarbonaté, prenant pour unité le lait normal, est de 85 après 24 heures de traite, et de 190 après 48 heures de traite $n$.

Il est donc nécessaire d'interdire la neutralisation aussi bien du lait entier que du lait centrifugé, celui-ci étant plus sensible encore.

Mais, pour réprimer cette falsification, il ešt indispensable de disposer de méthodes exactes pour déceler l'addition de carbonate et de bicarbonate de sodium.

Les méthodes anciennes sont peu précises; encore sont-elles faussées par le mouillage et par une fermentation lactique plus ou moins avancée.

Jadis, on décelait cette addition en faisant bouillir le lait et on concluait à la neutralisation lorsqu'on percevait une odeur de savon ; ou bien, on chauffait le lait plus longtemps ( 1 à 2 heures dans un courant de vapeur d'eau) et s'il était neutralisé, on remarquait que la coloration virait au jaune, puis au brun, et en cas d'excès de carbonate, il se produisait une mousse prolongée. Cela était évidemment peu précis.

Puis, on a préconisé (Schmidt et HILger) de mélanger $30 \mathrm{~cm}^{3}$ de lait à $250 \mathrm{~cm}^{3}$ d'eau, de coaguler à l'alcool et de constater ensuite la réaction alcaline du filtrat; on conseilla encore diverses réactions, celle par exemple au tanin (BOCHMEYeR) et à l'aspirine (LeLLI). On n'obtenait cependant pas encore une certitude suffisante.

On dosa ensuite (Soxhet-Schretbe), dans les cendres du lait, l'acide carbonique (sous forme de carbonate de calcium) qui se sépare quand on ajoute un excès d'eau de chaux à l'échantillon à 
examiner, la teneur normale de ces cendres étant de $2 \%$; cette méthode ne donna cependant pas non plus les résultats escomptés.

On s'arrêta longtemps à la détermination de l'alcalinité des cendres ; on traitait les cendres de $25 \mathrm{~cm}^{3}$ de lait avec $20 \mathrm{~cm}^{3}$ d'acide sulfurique décinormal, on chauffait 15 minutes au bain-marie pour chasser l'anhydride carbonique et on titrait à rebours avec la soude caustique décinormale.

Les cendres de lait normal ne sont que légèrement alcalines, en moyenne environ $2 \mathrm{gr}$. exprimés en carbonate de soude anhydre pour $100 \mathrm{gr}$. de cendres, alors que dans les laits neutralisés on atteint 7 gr., 8 gr. et même 11 à 13 grammes.

Mais on s'aperçut qu'on ne retrouvait pas dans les cendres l'alcalinité introduite; en effet, le bicarbonate, par exemple, agit sur les phosphates de calcium et de magnésium et donne des carbonates insolubles, et non alealins, tandis qu'il se forme du phosphate de soude soluble qui n'a pas de réaction alcaline non plus. On en vint donc à modifier cette méthode en dosant le phosphate de soude dans la partie soluble des cendres; le phosphate de soude, en effet, n'existe qu'en traces dans les cendres d'un lait normal, mais en plus forte quantité dans celles d'un lait neutralisé. On opère comme suit, : on incinère l'extrait de $25 \mathrm{~cm}^{3}$ de lait, on extrait à l'eau la partie soluble des cendres dans laquelle on détermine les alcalis libres par l'acide sulfurique décinormal, puis dans le même liquide le phosphate de soude par le nitrate d'urane, ou bien encore, en titrant d'abord à la phénolphtaléine et ensuite au méthylorange, mais dans ce cas on est obligé, pour être exact, de déduire $0 \mathrm{~cm}^{3} 7$ du résultat du premier titrage.

Mais l'expérience a démontré que cette méthode par détermination de l'alcalinité des cendres et les modifications y apportées ne permettent pas de retrouver avec grande certitude l'addition de petites quantités de carbonate ou de bicarbonate de sodium.

On a essayé aussi les indicateurs d'acidité, l'alizarine par exemple, l'acide rosolique, la galléine, le rouge de crésol, le rouge de phénol, le bleu de bromothy mol, la phénolphtaléine, etc. ; $1 \mathrm{~cm}^{3}$ de rouge de phénol à $0,2 \%$ est l'indicateur le plus sensible virant dans $10 \mathrm{~cm}^{3} \mathrm{de}$ lait du jaune rouge au rouge en présence de $0,059 \%$ de carbonate de soude; l'alizarine aussi peut donner des résultats relativement satisfaisants.

On a préconisé encore la conductivité électrique (STROHEOKER) et la cryoscopie (KoEnIG et KLUGE), le lactate de soude formé abaissant le point de congélation au-dessous de la limite 0,554.

Devant l'imperfection de toutes ces méthodes, le Professeur A. J. J. VANDEVELDE conseilla de déterminer dans les cendres du lait le rapport entre la teneur en métaux alcalins et la teneur en 
acide phosphorique, teneurs qui, dans le lait normal, sont sensiblement égales : $26,4 \%$ environ de sodium et potassium pour $26,3 \%$ d'anhydride phosphorique ; on incinère l'extrait de $250 \mathrm{~cm}^{3}$ de lait, on dissout les cendres dans un peu d'acide nitrique, on étend la solution à $100 \mathrm{~cm}^{3}$ et, dans une partie de celle-ci, on dose l'acide phosphorique, puis, dans une autre, les métaux alcalins, après élimination des phosphates par l'eau de baryte, des sels de calcium et de magnésium par le carbonate d'ammonium et ensuite par l'oxalate d'ammonium. Comme l'introduction d'un carbonate alcalin augmente la teneur des cendres en métaux alcalins et diminue leur teneur centésimale en acide phosphorique, on conclut à la neutralisation en présence de résultats comme les suivants :

$\begin{array}{llllll}\text { Métaux alcalins caleulés en sodium . . . } & 35,6 & 31,1 & 34,5 & 37,7 \\ \text { Anhydride phosphorique } \quad \ldots \ldots \ldots \ldots & 15,8 & 19,8 & 17,3 & 15,5\end{array}$

La méthode est fort longue, délicate à effectuer ; elle a le grand mérite d'avoir tracé la voie à la méthode indiscutablement la plus exacte : la détermination directe de la teneur en sodium des cendres, rendue possible par l'emploi du réactif de Blanchetière. C'est de cette méthode que je voudrais parler plus spécialement, parce que je la considère comme la plus exacte, capable de nous rendre les plus grands services pour l'identification et la répression de la falsification du lait par neutralisation. Elle permet, en effet, de doser gravimétriquement, volumétriquement et colorimétriquement des quantités de $1 \mathrm{mgr}$. de sodium avec une précision de $1 \%$.

Je rappelle que ce dosage du sodium repose sur la formation du sel de Streng, acétate triple d'uranyle, de magnésium et de sodium:

$$
\mathrm{NaC}^{2} \mathrm{H}^{3} \mathrm{O}^{2} . \mathrm{Mg}\left(\mathrm{C}^{2} \mathrm{H}^{3} \mathrm{O}^{2}\right)^{2} .3 \mathrm{UrO}^{2} .6,5 \mathrm{H}^{2} \mathrm{O}
$$

précipité par le réactif de Blanchetik̀ne contenant de l'acétate d'urane et de l'acétate de magnésium en solution aqueuse légèrement acétique.

Ce réactif a été modifié par KoLтhоFF qui a remplacé l'acétate de magnésium par l'acétate de zinc ; le sodium est précipité à l'état d'acétate triple d'uranyle, de zinc et de sodium

$$
\left.\mathrm{NaZn}\left(\mathrm{C}^{2} \mathrm{H}^{3} \mathrm{O}^{2}\right)^{3} \text {. (Ur } \mathrm{O}^{2}\right)^{3} \cdot 6 \mathrm{H}^{2} \mathrm{O}
$$

Chang et Tseng remplacent le sel de magnésium par celui de manganèse.

KAHANE, d'autre part, prépare un réactif hydro-alcoolique, de façon à abaisser la solubilité de l'acétate triple et à accélérer la précipitation.

Dans $100 \mathrm{em}^{3}$ de lait pur, la teneur en sodium est de $42 \mathrm{mgr}$. environ (42 mgr. 6 est une moyenne de 70 analyses de lait des envi- 
rons de Gand) ; et on peut considérer que tout lait, dans lequel cette teneur dépasse $50 \mathrm{mgr}$. pour $100 \mathrm{~cm}^{3}$, doit être déclaré additionné d'un sel de sodium.

Pour ce dosage, il y a lieu cependant de tenir compte que le potassium et les phosphates peuvent précipiter en même temps que le sodium par le réactif de BLANCHETIÈRE.

Le potassium ne précipite cependant qu'en grande quantité, au delà de $50 \mathrm{mgr}$. Nous ne devons donc pas en tenir compte, les cendres du lait normal ne contiennent, en effet, que $20 \%$ environ de potassium, et $10 \mathrm{~cm}^{3}$ mis en ouvre, par exemple, n'en contiendront que 15 mgr. environ, ce qui est à négliger.

Mais les phosphates sont à éliminer eomplètement. Cette élimination peut se faire de différentes façons : à l'acétate d'uranyle, au lait de chaux, au chlorure de baryum, ou sous forme de phosphate ammoniaco-magnésien, ou de phosphates de plomb ou de zine.

Quand on emploie le réactif-alcoolique de KAHANE, on obtient des résultats trop élevés si l'on sépare l'acide phosphorique au moyen de chlorure de baryum ou d'un sel de calcium, en raison de l'insolubilité de ces métaux dans l'alcool ; en présence de chlorure de baryum, on obtient des résultats qui sont de 3 à $10 \%$ trop élevés.

Tenant compte de ces conditions, on peut opérer des deux façons suivantes :

A. Précipiter la caséine de $100 \mathrm{~cm}^{3}$ de lait par l'acide chlorhydrique à froid ; filtrer et neutraliser l'excès d'acide du filtrat par l'a mmoniaque ; aciduler par un peu d'acide acétique, précipiter les phosphates par l'acétate d'uranyle, filtrer et prélever la quantité nécessaire pour le dosage du sodium.

Ou bien :

B. Evaporer la quantité déterminée de lait (5 à $10 \mathrm{~cm}^{3}$ ), l'incinérer jusqu'à obtention de cendres bien blanches et éliminer les phosphates des cendres de la façon suivante : les cendres de lait sont traitées au bain-marie, par une solution alcoolique d'acétate de magnésium à $2,5 \%\left(25 \mathrm{~cm}^{3}\right)$, on éva pore à sec et on incinère au rouge sombre. Les cendres ainsi obtenues sont dissoutes à chaud dans $4 \mathrm{~cm}^{3}$ d'acide chlorhydrique à $25 \%$; après refroidissement, on ajoute $10 \mathrm{~cm}^{3}$ d'ammoniaque, en remuant. Le phosphate ammoniacomagnésium est filtré et lavé à l'ammoniaque diluée. Le filtrat et les eaux de lavage réunis sont ensuite évaporés à sec au bain-marie et le résidu ešt repris par aussi peu d'eau distillée que possible, au maximum $5 \mathrm{~cm}^{3}$, qu'on additionne de la quantité nécessaire (10 fois au moins) de réactif de Blanchetière. On peut aussi, sans passer par l'addition d'acétate de magnésium, précipiter directement les phos- 
phates dans la solution des cendres en acide chlorhydrique. C'est cette dernière méthode qui m'a donné le meilleur résultat, en opérant sur $10 \mathrm{~cm}^{3}$ de lait normal ou sur $5 \mathrm{~cm}^{3}$ de lait neutralisé.

Pour le dosage proprement dit du sodium, on peut opérer gravimétriquement - ce qui est le plus rapide et suffisamment précis - en pesant le précipité d'acétate triple obtenu dont le poids multiplié par 0,0153 (réactif de BLANCHetière) ou par 0,01495 (réactif modifié par KolthofF) donne la teneur en sodium. Si on a employé le réactif modifié par KoLthoFf, pour $10 \mathrm{~cm}^{3}$ de lait pur mis en œuvre, le poids du précipité d'acétate triple est de $280 \mathrm{mgr}$. entiron.

Une addition de $0 \mathrm{gr}$. 5 au litre de carbonate de soude pur et anhydre, porte le poids de ce précipité à 426 gr. environ ; une addition de $1 \%$, à $571 \mathrm{mgr}$. environ. Le précipité d'acétate triple pèse à peu près 67 fois plus que la quantité de sodium qu'il renferme ; cela rend la méthode extrêmement sensible et permet de doser avec exactitude des quantités très minimes de sodium.

On peut également opérer colorimétriquement en dissolvant le précipité dans de l'eau à $60^{\circ} \mathrm{C}$. et en comparant l'intensité de coloration avec celle d'une solution type préparée avec 2 gr. 242 de chlorure de sodium par litre; on peut aussi additionner cette solution d'une solution de ferrocyanure de potassium et mesurer le coefficient d'extinction au colorimètre absolu de LEITZ, étalonné avec des solutions à titre connu.

On peut encore effectuer le dosage volumétriquement en réduisant le sel uranique jaune en sel uraneux vert par l'acide sulfurique, en présence de zinc ou de cuivre et en réoxydant ce dernier par une solution titrée de permanganate. On connaît aussi une méthode iodométrique.

Enfin, on peut encore doser le sodium dans le précipité en déterminant la quantité d'uranium qu'il contient, l'acétate triple de magnésium, d'uranium et sodium en contenant $53,79 \%$.

Ce dosage du sodium appliqué au lait est donc parfaitement mis au point et permet, par conséquent, de déceler avec certitude l'addition de quantités minimes de carbonate ou bicarbonate de sodium.

Mais il constitue essentiellement une méthode de laboratoire relativement longue et non une méthode de détection.

Pour cela, nous avons à notre disposition l'excellente méthode de Eble et Pfeiffer. Elle consiste à additionner $20 \mathrm{~cm}^{3}$ de lait de $30 \mathrm{~cm}^{3}$ d'alcool méthylique pur et à agiter le mélange brusquement à trois reprises ; on observe alors, dans le cas d'un lait pur, une coagulation des albumines en masses importantes laissant apparaître rapidement un sérum elair dont l'acidité libre est comprise entre les 
$p \mathrm{H} 6,3$ et 6,5 ; en cas de lait neutralisé, on ne constate au contraire aucune coagulation, la masse reste homogène pendant plusieurs jours et, par filtration, on ne peut obtenir qu'un sérum trouble et laiteux dont le $p \mathrm{H}$ s'élève au-dessus de 6,5 et pouvant même atteindre 8,5 en cas d'une addition relativement forte de carbonate ou bicarbonate de sodium. Cette méthode que j'ai eu l'occasion d'expérimenter sur un grand nombre d'échantillons de lait donne d'excellents résultats, mais seulement au point de vue de la détection des laits neutralisés.

La formation du coagulum peut cependant, dans certains cas, provoquer un doute. En effet, dans un lait fraîchement trait, la coagulation est ténue et elle augmente à mesure que l'acidité croît, car elle est liée à la présence d'acides libres (acide carbonique et acide lactique) ; et, pour ne pas se produire dans un lait carbonaté, il faut que l'alealinisation ait été suffisante pour neutraliser cette acidité, la présence de faibles quantités d'acides libres permet encore une coagulation partielle. Mais la détermination de l'acidité libre de sérum permet de réduire ee doute à néant, un $p H$ plus élevé que 6,5 étant toujours un indice de neutralisation ; par exemple, le sérum méthylique d'un lait additionné de 0 gr. 2 par litre de carbonate de sodium accuse un $p \mathrm{H}$ de 6,8 ; si on désire opérer rapidement, on peut déterminer cette acidité libre à l'aide de l'indicateur universel de Merck.

Nous avons donc ainsi, à notre disposition, une méthode excellente de détection et une autre d'analyse qui nous permettent de retrouver avec certitude la moindre addition de carbonate ou de bicarbonate de sodium au lait.

\title{
REVUE
}

\section{ENTRETIEN ET NETTOYAGE DES MACHINES A TRAIRE}

par

\author{
G. GÉNIN
}

Ingénieur chimiste E. P. C.

L'emploi des machines à traire n'a pas encore fait de très grands progrès en France, alors qu'aux Etats-Unis, en Australie, en Nouvelle-Zélande et même en Grande-Bretagne, ces machines sont très répandues dans le monde agricole. C'est ainsi qu'en Nouvelle Zélandec il a été établi que sur 4 fermes, 3 sont munies de machines à traire.

Il faut néanmoins espérer que l'emploi de ces machines ira en se 